\title{
Molecular Imprinting with Ruthenium Porphyrin Catalysts
}

\author{
Estelle Burri§ and Kay Severin* \\ \$METTLER TOLEDO Award Winner (Oral Presentation)
}

\begin{abstract}
A ruthenium meso-tetra[4-(vinylbenzoxy)benzyl]porphyrin catalyst with four polymerizable side chains was synthesized in three steps from commercially available starting materials. The polymerizable side chains allowed the incorporation of this complex in highly cross-linked mesoporous co-polymers. A block polymer and a molecularly imprinted polymer were prepared by co-polymerization with ethyleneglycol dimethacrylate using chloroform as the porogen. The imprinted polymer was prepared in the presence of aminodiphenylmethane as a template. Subsequent removal of the template created a substrate pocket next to the catalytically active center. Both polymers displayed a high catalytic activity for the oxidation of alcohols and alkanes with 2,6-dichloropyridine- $\mathrm{N}$-oxide as the oxidant while the homogeneous catalyst was completely inactive. Furthermore, the imprinted polymer displayed a significant increase in activity compared to the block co-polymer.
\end{abstract}

Keywords: Catalyst $\cdot$ Molecular imprinting $\cdot$ Porphyrin $\cdot$ Ruthenium

\section{Introduction}

The interest in ruthenium porphyrin chemistry was inspired by both the periodic relationship of ruthenium to iron - and hence of ruthenium porphyrins to hemoproteins - and the possibility to prepare relatively stable oxoruthenium derivatives. Most work in this context has focused on oxidation reactions [1] but some research has also been directed towards other reactions, such as the cyclopropanation of olefins [2], the aziridination of alkenes [1b][2e][3], the carbonyl ylide/1,3-dipolar cycloaddition reactions of $\alpha$-diazoketones [4], the insertion of carbenes into the $\mathrm{S}-\mathrm{H}$ bonds [5], and the olefination of aldehydes [6].
The design of heterogeneous ruthenium porphyrin catalysts for these reactions was achieved by various methods: by encapsulation in mesoporous molecular sieves [7] or sol-gel silica [8], by electropolymerization of complexes with fluorene groups [9], by covalent attachment to Merrifield resins [10] or by co-polymerization of the ruthenium porphyrin catalyst with an excess of ethyleneglycol dimethacrylate (EGDMA) [11]. These supported catalysts showed good catalytic activities, but leaching problems were encountered in some cases [7b][8].

In the following, we describe a polyEGDMA supported ruthenium porphyrin catalyst, which was prepared by molecular imprinting [12]. This imprinted polymer proved to be a very efficient catalyst for the oxidation of secondary alcohols and alkanes with 2,6-dichloropyridine $\mathrm{N}$-oxide $\left(\mathrm{Cl}_{2}\right.$ pyNO$)$ as the oxidant [13].

\section{Results}

The carbonyl ruthenium meso-tetra[4(vinylbenzoxy)benzyl]porphyrin complex 1 was synthesized in three steps starting from commercially available products [13]. First, 4-(vinylbenzoxy)benzaldehyde was prepared by reaction of $p$-hydroxybenzaldehyde with $p$-vinylbenzylchloride [14]. The aldehyde was then re- acted with pyrrole to form the porphyrin macrocycle following the standard AdlerLongo procedure [15]. Finally, the metal was inserted in the porphyrin to give complex 1 (Fig. 1). The insertion reaction with $\left[\mathrm{Ru}_{3}(\mathrm{CO})_{12}\right]$ was done in the presence of styrene, which acts as a hydrogen scavenger and prevents the reduction of the polymerizable vinyl groups of the porphyrin [11]. The metallomonomer 1 was subsequently co-polymerized with EGDMA $(\mathbf{1}: \mathrm{EGDMA}=1: 400)$ in the presence of 1 equiv. of aminodiphenylmethane which serves as a template for the imprinting procedure and with chloroform as the porogen (Scheme 1). The resulting dark-red polymer was ground in a mortar and then washed with a solution of trifluoroacetic acid in acetone to remove the pseudosubstrate. This washing procedure yields the imprinted polymer P1 with substrate pockets next to the catalytically active ruthenium centers [13]. A control polymer $\mathbf{P 2}$ was prepared in the same fashion but without the pseudo-substrate (Fig. 2). Hence, this polymer $\mathbf{P 2}$ has an identical first coordination sphere but it does not have substrate pockets. For the imprinted polymer P1, a BET surface area of $357 \mathrm{~m}^{2} /$ $\mathrm{g}$ and an average pore size of $58 \AA$ were determined. For the control polymer P2, a slightly larger surface area $\left(409 \mathrm{~m}^{2} / \mathrm{g}\right)$ was measured while the average pore size was determined to be slightly smaller ( 54 
$\AA$ ). These measurements confirmed the porosity of both polymers and therefore the accessibility of the catalytically active ruthenium centers.

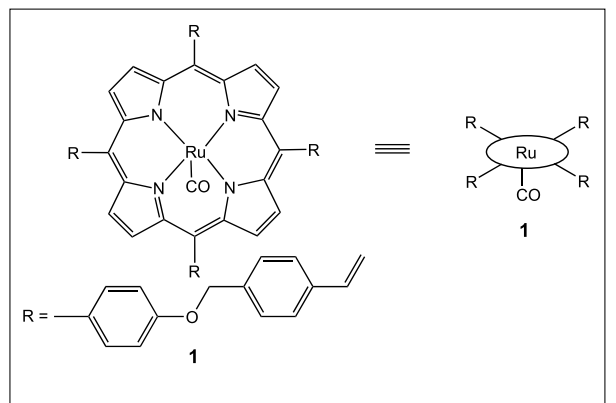

Fig. 1. Ruthenium meso-tetra[4-(vinylbenzoxy)benzyl]porphyrin 1

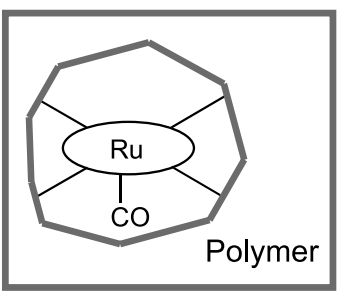

P2

Fig. 2. Control polymer P2

The catalytic activity of the heterogeneous (P1 and P2) and the homogeneous (1) catalysts was then assessed. A comparison of the catalytic activity of complex 1, control polymer $\mathbf{P 2}$ and imprinted polymer P1 was made for the oxidation of diphenylmethane to benzophenone with 2,6-dichloropyridine-N-oxide as the oxidant (Scheme 2, Fig. 3). The homogeneous catalyst 1 showed no activity, which was expected since it was reported that the addition of a strong mineral acid such as $\mathrm{HBr}$ is essential to perform this reaction [16]. P2 was moderately active and $\mathbf{P 1}$ displayed the highest activity. The increase of activity of polymer $\mathbf{P 2}$ compared to the homogeneous catalyst 1 can be explained by a site-isolation effect, which prevents the irreversible dimerisation of the ruthenium porphyrin catalysts upon oxidation. The further increase of activity displayed by the imprinted polymer $\mathbf{P 1}$ can be attributed to the free substrate pocket next to the active site, which allows a better access of the substrates to the ruthenium center. This increase in activity is remarkable since the ruthenium complexes of the control polymer $\mathbf{P 2}$ and the imprinted polymer $\mathbf{P 1}$ have an identical first coordination sphere.

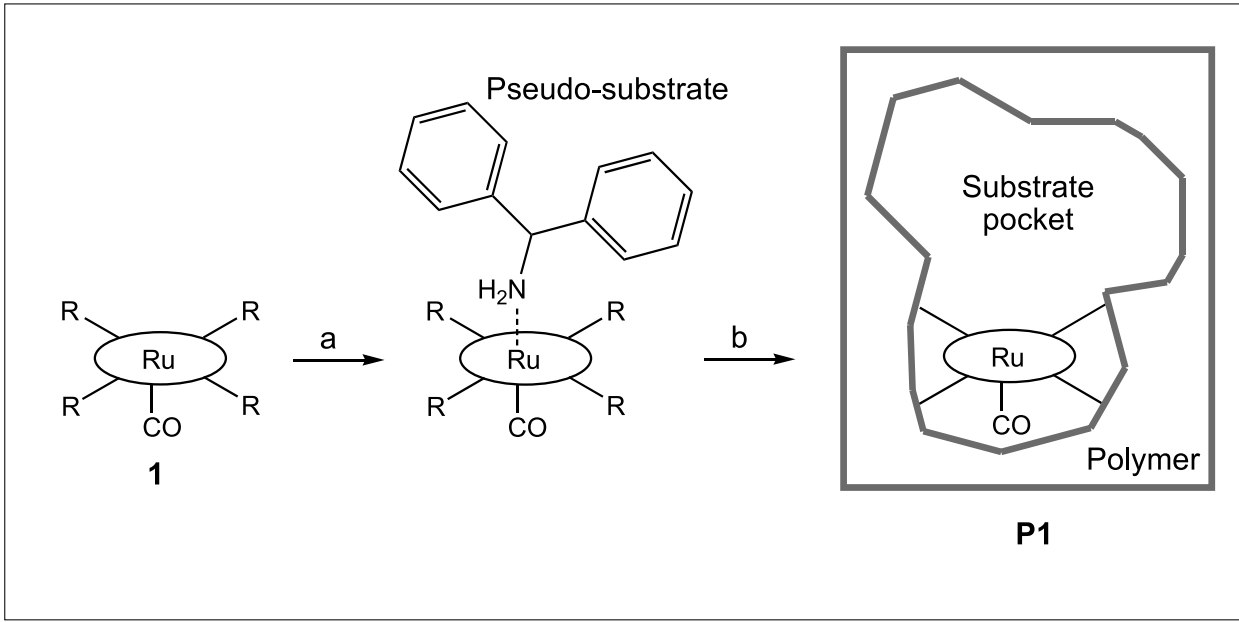

Scheme 1. Preparation of molecularly imprinted polymer P1. a) Addition of the pseudo-substrate; b) co-polymerization with EDGMA followed by the pseudo-substrate removal.

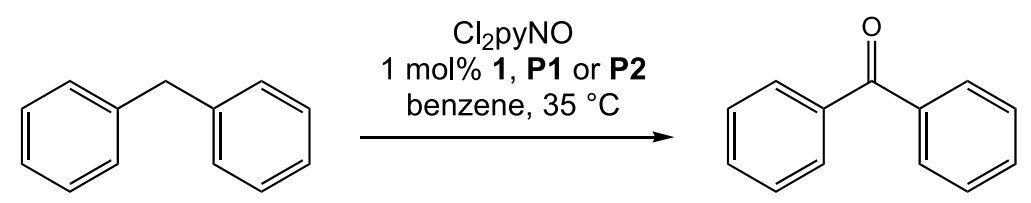

Scheme 2. Oxidation of diphenylmethane by $\mathrm{Cl}_{2}$ pyNO with the homogeneous catalyst 1, the control polymer P2, and the imprinted polymer P1

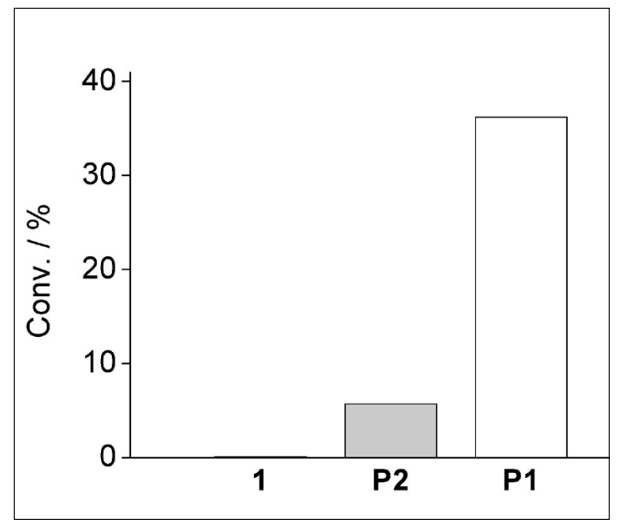

Fig. 3. Oxidation of diphenylmethane by $\mathrm{Cl}_{2}$ pyNO with the homogeneous catalyst $\mathbf{1}$, the control polymer P2, and the imprinted polymer $\mathbf{P 1}$. The reactions were performed in benzene at $35{ }^{\circ} \mathrm{C}$ with a substrate $/ \mathrm{Cl}_{2}$ pyNO/catalyst molar ratio of $100: 250: 1$. The values were measured by GC chromatography after $6 \mathrm{~h}$.

Polymer P1 was further tested for the oxidation of various alkanes (Table). Moderate to excellent yields were obtained with these difficult substrates. For the homogeneous catalyst $\mathbf{1}$, on the other hand, negligible conversions (less than $2 \%$ ) were recorded under the same conditions.

Substrate selectivity was also investigated with the imprinted polymer P1 and
Table. Catalytic oxidation of alkanes with the imprinted catalyst $\mathbf{P} \mathbf{1}^{\mathrm{a}}$

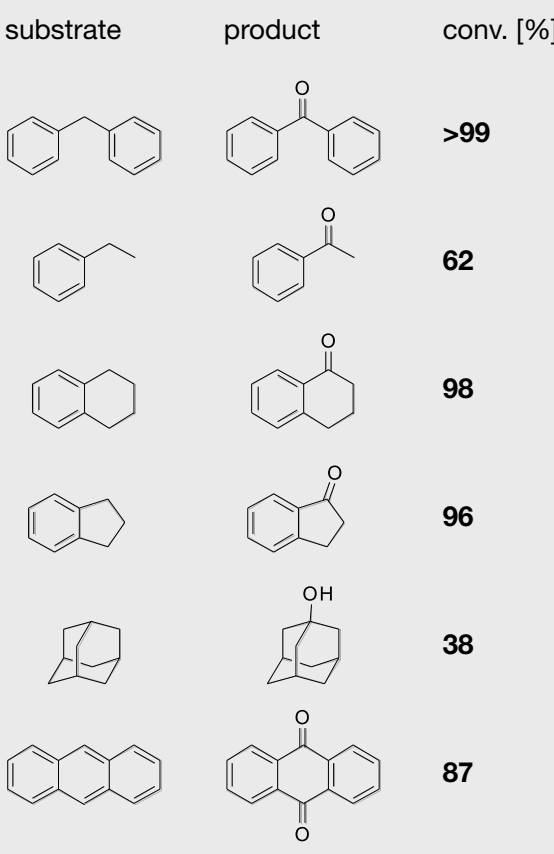

aThe reactions were performed in benzene at $55{ }^{\circ} \mathrm{C}$ with a substrate $/ \mathrm{Cl}_{2}$ pyNO/catalyst molar ratio of 100:250:1 (100:500:1 for anthracene). The conversion was determined after $24 \mathrm{~h}$ by gas chromatography. 
a second imprinted polymer $\mathbf{P 3}$, prepared following the same protocol but using 1adamantylamine as the template instead of diphenylmethylamine. For this experiment, two different substrates (in the same reaction vessel) were oxidized at the same time by the respective imprinted polymer $\mathbf{P 1}$ or $\mathbf{P 3}$ or the control polymer P2. With the imprinted polymer P1, no selectivity was found. With the imprinted polymer P3, however, a substrate selectivity of 1.4 in favor of 2-adamantanol was observed for the oxidation of 2-adamantanol in competition with 2-octanol (Fig. 4). The relatively low selectivity compared to other imprinted metal catalysts [17] can be explained by the single bond which links template and catalyst during the polymerization: rotational freedom around this bond yields a larger, less defined substrate pocket. The incorporation of secondary interaction between the template and the polymer could help to overcome this drawback.

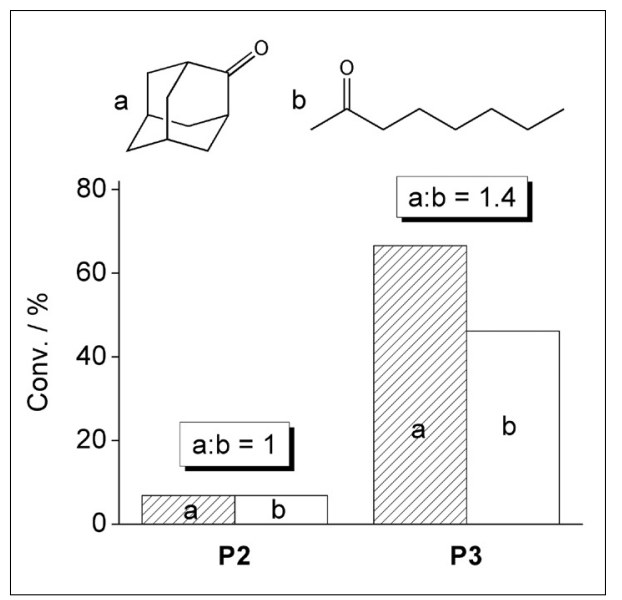

Fig. 4. Competitive oxidation of a mixture of 2-adamantanol and 2-octanol catalyzed by either the imprinted polymer P3 or by the control polymer P2. A selectivity of 1.4 in favor of adamantanone was observed with the imprinted polymer P3. The reactions were performed in benzene at room temperature with a 2-adamantanol/2-octanol/ $\mathrm{Cl}_{2}$ pyNO/catalyst molar ratio of 100:100:250:1. The values were measured by GC chromatography after $4 \mathrm{~h}$.

\section{Conclusions}

A ruthenium meso-tetra[4-(vinylbenzoxy)benzyl]porphyrin complex was successfully incorporated in a mesoporous, highly cross-linked EDGMA polymer. The use of a template during the polymerization allowed the creation of a substrate pocket next to the catalytically active site by molecular imprinting. A control polymer was prepared following the same protocol but without template. Both polymers showed a high catalytic activity for the oxidation of alcohols and alkanes while the corresponding homogeneous ruthenium porphyrin complex was inactive under the conditions employed. The enhanced reaction rates displayed by the control polymer can be attributed to site-isolation effects. The even larger increase in activity displayed by the imprinted polymer can be attributed to the substrate pocket, which allows a better access of the substrate to the active ruthenium center. The difference in activity between the two polymers of approximately one order of magnitude is impressive given that both polymers contain the same amount of ruthenium catalyst having an identical first coordination sphere. Highly cross-linked organic polymers therefore represent ideal supports for supported Ru-porphyrin catalysts: they not only prevent intermolecular deactivation reactions but also allow the manipulation of the microenvironment of the catalyst in a controlled fashion.

Received: February 19, 2006

[1] a) B. Meunier, Chem. Rev. 1992, 92, 1411-1456; b) C.-M. Che, W.-Y. Yu, Pure Appl. Chem. 1999, 71, 281-288; c) J.T. Groves, K. Shalyaev, J. Lee, in 'The Porphyrin Handbook', Vol. 4, Eds.: K.M. Kadish, K.M. Smith, R. Guilard, Academic Press, New York, 2000, pp. 17-40; d) G. Simonneaux, P. Le Maux, Coord. Chem. Rev. 2002, 228, 43-60.

[2] a) E. Galardon, P. Le Maux, G. Simonneaux, Chem. Commun. 1997, 927-928; b) M. Frauenkron, A. Berkessel, Tetrahedron Lett. 1997, 38, 7175-7176; c) E. Galardon, P.L. Maux, L. Toupet, G. Simonneaux, Organometallics 1998, 17, 565-569; d) E. Galardon, P. Le Maux, G. Simonneaux, Tetrahedron 2000, 56, 615-621; e) J.-L. Zhang, C.-M. Che, Org. Lett. 2002, 4, 1911-1914; f) Y. Li, J.-S Huang, G.-B. Xu, N. Zhu, Z.-Y. Zhou, C.-M. Che, K.-Y. Wong, Chem. Eur. J. 2004, 10, 3486-3502.

[3] J.-L. Liang, J.-S. Huang, X.-Q. Yu, N. Zhu, C.-M. Che, Chem. Eur. J. 2002, 8 , 1563-1572.

[4] C.-Y. Zhou, W.-Y. Yu, C.-M. Che, Org. Lett. 2002, 4, 3235-3238.

[5] a) E. Galardon, P. Le Maux, G. Simonneaux, J. Chem. Soc., Perkin Trans. 1 1997, 2455-2456; b) E. Galardon, S. Roue, P. Le Maux, G. Simonneaux, Tetrahedron Lett. 1998, 39, 2333-2334.

[6] Y. Chen, L. Huang, M.A. Ranade, X.P. Zhang, J. Org. Chem. 2003, 68, 37143717.

[7] a) J.-L. Zhang, Y.-L. Liu, C.-M. Che, Chem. Commun. 2002, 2906-2907; b) C.-J. Liu, W.-Y. Yu, S.-G. Li, C.-M. Che, J. Org. Chem. 1998, 63, 7364-7369; c) C.-J. Liu, S.-G. Li, W.-Q. Pang, C.-M. Che, Chem. Commun. 1997, 65-66.
[8] R. Zhang, W.-Y. Yu, K.-Y. Wong, C.-M. Che, J. Org. Chem. 2001, 66, 8145-8153.

[9] C. Paul-Roth, J. Rault-Berthelot, G. Simonneaux, Tetrahedron 2004, 60, 1216912175.

[10] X.-Q. Yu, J.-S. Huang, W.-Y. Yu, C.-M. Che, J. Am. Chem. Soc. 2000, 122, 53375342.

[11] O. Nestler, K. Severin, Org. Lett. 2001, 3, 3907-3909.

[12] a) M. Yan, O. Ramströhm (Eds), 'Moleculary Imprinted Materials: Science and Technology', Marcel Dekker, New York, 2005; b) B. Sellergren (Ed.), 'Molecularly Imprinted Polymers: Man-Made Mimics of Antibodies and Their Applications in Analytical Chemistry', Elsevier Science B.V., Amsterdam, 2001; c) J.J. Becker, M.R. Gagné, Acc. Chem. Res. 2004, 37, 798-804; d) S. Striegler, J. Chromatogr. B 2004, 804, 183-195; e) M. Tada, Y. Iwasawa, J. Mol. Catal. A 2003, 199, 115-137; f) C. Alexander, L. Davidson, W. Hayes, Tetrahedron 2003, 59, 2025-2057; g) G. Wulff, Chem. Rev. 2002, 102, 1-27; h) K. Severin, Curr. Opin. Chem. Biol. 2000, 4 , 710-714.

[13] E. Burri, M. Ohm, C. Daguenet, K. Severin, Chem. Eur. J. 2005, 11, 5055-5061.

[14] H. Kamogawa, Y. Haramoto, M. Nanasawa, Bull. Chem. Soc. Jpn. 1979, 52, 846848.

[15] A.D. Adler, F.R. Longo, J.D. Finarelli, J. Goldmacher, J. Assour, L. Korsakoff, $J$. Org. Chem. 1967, 32, 476.

[16] H. Ohtake, T. Higuchi, M. Hirobe, J. Am. Chem. Soc. 1992, 114, 10660-10662.

[17] K. Polborn, K. Severin, Chem. Eur. J. 2000, 6, 4604-4611. 